\title{
Inclusão digital: resultados de um curso de informática
} para idosos

\section{Digital inclusion: results of a computer course for elderly}

\author{
José Raphael Bokehi ${ }^{1}$, Gian Vitor Almeida Rocha ${ }^{2}$, Maria Carmen Vilas-Bôas Hacker Alvarenga ${ }^{3}$ \\ 1 Professor. Doutor. Universidade Federal Fluminense (UFF), Brasil. E-mail: jraphael@ic.uff.br \\ 2 Acadêmico. Universidade Federal Fluminense (UFF), Brasil. E-mail: gianr@id.uff.br \\ 3 Assistente Social. Mestre. Universidade Federal Fluminense (UFF), Brasil. E-mail: mcarmenvbhalvarenga@gmail.com
}

Recebido em: 19/01/2020 | Aprovado em: 12/03/2020

DOI: $10.12957 /$ interag.2019.53548

\begin{abstract}
Resumo
A inclusão digital de idosos ainda representa um desafio no Brasil. O Projeto Incluir surgiu em 2006, sob o financiamento da Financiadora de Estudos e Projetos (FINEP), visando atender ao Pré-Universitário Popular da Escola de Engenharia da UFF. Em 2007, ampliou sua atuação ao público idoso através da parceria com o do programa de extensão Espaço Avançado da Terceira Idade, da Escola de Serviço Social da UFF, um dos primeiros programas institucionais a focalizar questões de envelhecimento e $a$ atender à terceira idade em Niterói. $O$ papel do Projeto Incluir é prover o acesso à tecnologia a uma parcela da população que, por motivos culturais ou socioeconômicos, se vê à parte do mundo informatizado. Este trabalho relata os resultados obtidos por este projeto no período de 2010 a 2018. Para tanto foi efetuada uma busca documental nos relatórios anuais do projeto, bem como nas avaliações dos bolsistas e alunos que dele participaram neste mesmo período. Foram atendidos 384 idosos, em turmas de nível inicial e avançado. 0 projeto contou com 20 bolsistas de extensão, 5 discentes voluntários e 2 professores ao longo de seu funcionamento. As avaliações dos alunos mostram satisfação com o curso e incorporação de atividades ligadas à informática em suas atividades de rotina. Dessa forma, conclui-se que o projeto vem contribuindo para ampliar a utilização das ferramentas de informática no cotidiano dos alunos, favorecendo a adoção de novas formas de inserção social e o uso de ferramentas de interesse na sua rotina.
\end{abstract}

Palavras-chave: Inclusão Digital. Idosos. Extensão Universitária

\begin{abstract}
Digital inclusion of the elderly still represents a challenge in Brazil. The Include Project came into being in 2006, under the funding of Financiadora de Estudos e Projetos (FINEP), aimed at serving the Popular Pre-University of the UFF School of Engineering. In 2007, it expanded its activities to the elderly public through its partnership with the Advanced Space for Seniors extension program of the UFF School of Social Service, one of the first institutional programs to focus on aging issues and to assist the elderly in Niterói. The role of the Include project in this partnership is to respond to the need to provide access to technology for a portion of the population who, for cultural or socio-economic reasons, find themselves apart from the computerized world. This paper reports the results obtained by this project in the period from 2010 to 2018. To this end, a documentary search was made in the project's annual reports, as well as in the evaluations of the fellows and students who participated in the project in this same period. A total of 384 seniors were attended, in initial and advanced level classes. The project had 20 extension scholars, 5 volunteer students and 2 teachers throughout its operation. Student evaluations show satisfaction with the course and incorporation of computer related activities into their routine activities. Thus, it is concluded that the project has been meeting its objectives.
\end{abstract}

Keywords: Digital Inclusion. Seniors. University Extension

Área temática: Educação.

Linha de extensão: Terceira Idade. 


\section{Introdução}

Segundo o Instituto Brasileiro de Geografia e Estatística (IBGE) ${ }^{1}$, a composição populacional por grupos de idade no Brasil mostra tendência de envelhecimento demográfico, ou seja, aumento percentual de adultos e idosos na população. Por outro lado, tem sido discutida a relevância de se buscar o chamado envelhecimento saudável e o papel que as redes sociais desempenham neste processo. ${ }^{24}$

Araújo e colaboradores ${ }^{2}$ apontam que o envelhecimento saudável deve ser analisado sob três aspectos: a menor probabilidade de adoecer, manutenção de alta capacidade física e mental, além do engajamento social ativo. Geib ${ }^{3}$ discute a influência das características do contexto social na saúde, ao longo da vida, as quais podem gerar desigualdades e vulnerabilidades que irão influenciar o bem-estar, a independência e a qualidade de vida dos idosos, destacando o papel da interação social e na comunidade nesse processo. Araújo e colaboradores² ${ }^{2}$ ressaltam a importância de se estimular a participação, a interação, a integração e o empoderamento e libertação dos idosos na melhoria de sua qualidade de vida.

As tecnologias da informação e da comunicação (TIC) são consideradas ferramentas importantíssimas para a promoção da inclusão do indivíduo na sociedade contemporânea uma vez que estas tecnologias invadiram diversos setores, tornando a sociedade informatizada. ${ }^{5 \cdot 7}$ Dados da Pesquisa Nacional por Amostra de Domicílios1, realizada pelo IBGE em 2017, mostram que $67,9 \%$ dos domicílios brasileiros têm acesso domiciliar à internet, sendo de $47,7 \%$ o percentual dos domicílios com rendimento domiciliar per capita abaixo de 5,5 dólares por dia que tem este mesmo acesso. Vale ressaltar, no entanto, que a exclusão digital é um processo complexo que não se limita ao acesso a computadores e à internet, pois envolve aspectos como a qualidade do acesso, capacidade de leitura e interpretação das informações, nível de escolaridade entre outros. ${ }^{8}$

Na medida em que a internet se tornou o mais amplo sistema de informação global, também se acentuou a chamada exclusão digital, fazendo com que pessoas com menor acesso a esta tecnologia fiquem à margem de diversos processos sociais. Os idosos estão entre as pessoas que, geralmente, são mais afetadas pela exclusão digital. ${ }^{7-14}$ Embora a inclusão digital de idosos tenha sido objeto de intensa discussão nas últimas décadas, ${ }^{5-15}$ ainda representa um desafio no Brasil..$^{5,6,14}$ 
Avaliando os conhecimentos e aptidões de 200 idosos no uso de tecnologias digitais, Lolli e Maio ${ }^{14}$ observaram que a maioria deles possuía computador e conhecimentos sobre esta ferramenta, reconhecendo que o uso da informática facilita as atividades diárias. Tezza e Bonia ${ }^{6}$ observaram que os idosos se sentem excluídos tanto no meio profissional quanto social e que a internet é percebida como um canal de aproximação com os filhos, além de permitir acesso à informação e a diversão. Oliveira ${ }^{15}$ destaca o papel do uso da internet, em especial de e-mail e grupos como Orkut e Facebook, na interação social dos idosos. A ampliação do contato social, a possibilidade de se manterem atualizados e de buscar novas oportunidades de trabalho foram algumas das motivações observadas por Batista e colaboradores ${ }^{16}$ ao estudarem o modo como os idosos que participam da Universidade Aberta à Terceira Idade da Universidade de São Paulo utilizam as tecnologias de informação e comunicação.

Estudo realizado em Florianópolis ${ }^{13}$ por pesquisadores da Universidade Federal de Santa Catarina, com adultos com 60 anos ou mais vivendo na área urbana, entrevistou, em 2009. 2010 um total de 1705 idosos, dos quais 1197 foram novamente entrevistados 4 anos mais tarde. Os resultados mostraram que apenas $26,6 \%$ dos entrevistados no segundo momento do estudo utilizavam a internet. Deles, $7.5 \%$ havia iniciado o uso nos 4 anos cobertos pelo estudo. Batista e colaboradores16 observaram heterogeneidade entre os idosos entrevistados quanto ao grau de independência e autonomia no uso de tecnologias de informação. Esta observação é reforçada por Grossi e colaboradores ${ }^{17}$ que apontam que ainda há carência de projetos e programas de inclusão digital no Brasil, de forma a contribuir para reduzir a desigualdade social no país.

É nesse contexto que se insere o projeto Incluir, cujo objetivo é, através da inclusão digital dos idosos, contribuir para sua socialização, num cenário em que a utilização da tecnologia da informação se torna cada vez mais disseminada e um elemento importante para o mercado de trabalho e a vida cotidiana dos idosos. Vale ressaltar que o projeto Incluir é parceiro do programa de extensão Espaço Avançado, um dos primeiros programas institucionais a focalizar questões de envelhecimento e a atender à terceira idade em Niterói. 


\section{Método}

O estudo envolveu a análise documental dos relatórios anuais do projeto e dos discentes do curso de Ciência da Computação que atuaram como bolsistas de extensão, registrados no Sistema de Informação e Gestão de Projetos (SIGPROJ), bem como das avaliações dos idosos que participaram do curso no período de 2010 a 2018. Os documentos foram analisados por duas pessoas de forma independente. Havendo discrepância entre as informações coletadas, um terceiro indivíduo buscava o dado nos documentos, visando dirimir a dúvida. As variáveis quantitativas consideradas foram o número de turmas oferecidas anualmente, o número de inscritos em cada turma, a frequência média dos alunos, a equipe do projeto a cada ano, os trabalhos apresentados e os prêmios obtidos. Estas variáveis foram analisadas a partir de ferramentas da estatística descritiva.

A partir do formulário de avaliação preenchido pelos idosos atendidos, foram identificadas as sugestões formuladas, bem como as críticas e percepção sobre o curso. A análise destes depoimentos se deu através da análise de conteúdo proposta por Bardin ${ }^{18}$, que propõe o uso de procedimentos sistemáticos e objetivos para a descrição do conteúdo das mensagens. Nessa análise, dois eixos foram considerados: a sensação dos alunos em relação ao curso e ao seu desempenho no uso da informática de forma que se pudesse investigar o impacto do curso na desenvoltura dos idosos com as ferramentas trabalhadas e com o processo propriamente dito. $\mathrm{O}$ software gratuito wordclouds, acessado online, também foi usado na avaliação das respostas dos alunos, a fim de captar as ideias centrais apresentadas.

\section{Resultados}

\section{A estrutura do curso}

"O curso se estrutura em 3 módulos: básico, avançado I e avançado II. No módulo básico, o foco está na ambientação com o hardware e software e em exercícios de digitação e planilhas, visando diminuir a resistência à utilização do equipamento e a dificuldade de executar/lembrar comandos e de usar o mouse, principais dificuldades expressas pelos idosos. No módulo avançado, inicia-se a interação com a internet, e no 
módulo avançado II, além de ser aprofundada a interação com a internet, são trabalhados também outros dispositivos, como smartphones. A principal dificuldade apresentada pelos alunos se refere à quantidade de funções que o computador apresenta e a falta de familiaridade com a simbologia usada na internet. Como as aulas são interativas, de acordo com as dúvidas e necessidades que os alunos vão expondo, a ementa pode sofrer modificações.

Cada módulo do curso tem a duração de um ano (maio até dezembro), com carga horária de 60 horas. As aulas, todas práticas, são ministradas uma vez por semana, com duração de 2 horas cada, sendo oferecidas às terças, quartas e quintas-feiras nos Laboratórios do Instituto de Computação. O curso usa como base a apostila criada especificamente para o curso pela equipe do projeto para trabalhar Informática Básica. Além do conteúdo teórico, possui também atividades propostas a serem realizadas no laboratório. Entre os temas que integram a apostila estão conceitos básicos de armazenamento de arquivos no computador, edição de textos e de imagens, planilhas, preparação de slides e navegação na internet. A inclusão de outros temas, como o uso de smartphones, por exemplo, teve por objetivo que os idosos pudessem se sentir ainda mais incluídos digitalmente.

A avaliação do desempenho do aluno é continuada (análise individual, reforçando o conteúdo nas dificuldades pessoais), e, no final do curso, há um exercício avaliativo, a fim de medir os conhecimentos adquiridos. Os alunos também são convidados a responder a um questionário de avaliação preenchido de forma anônima, após a conclusão das atividades. A distribuição de certificados de conclusão tem se mostrado um incentivo para que os alunos participem e prossigam nos outros módulos.

\section{Os resultados obtidos}

O projeto Incluir teve início em 2006. Numa primeira etapa, buscou-se identificar nos projetos parceiros situações em que a tecnologia pudesse ser utilizada ativamente pelo público alvo. Sobre estas atividades concretas foram planejados os 
conteúdos dos cursos e oficinas. No primeiro grupo, os temas trabalhados envolveram as ferramentas e componentes básicos do computador, editores de texto, planilhas e Internet. Assim, até hoje, a familiarização e o aprendizado da tecnologia decorrem do exercício prático atrelado a atividades do cotidiano do grupo. A cada turma são reavaliadas as atividades propostas, o que leva à atualização da apostila preparada especialmente para o curso.

A figura 1 apresenta o número de vagas e de inscritos ao longo do período de estudo. Ao todo, foram ofertadas 600 vagas, das quais 384 (64\%) foram ocupadas. A média anual de inscritos foi de 39 idosos $(\mathrm{dp}=27,11)$.

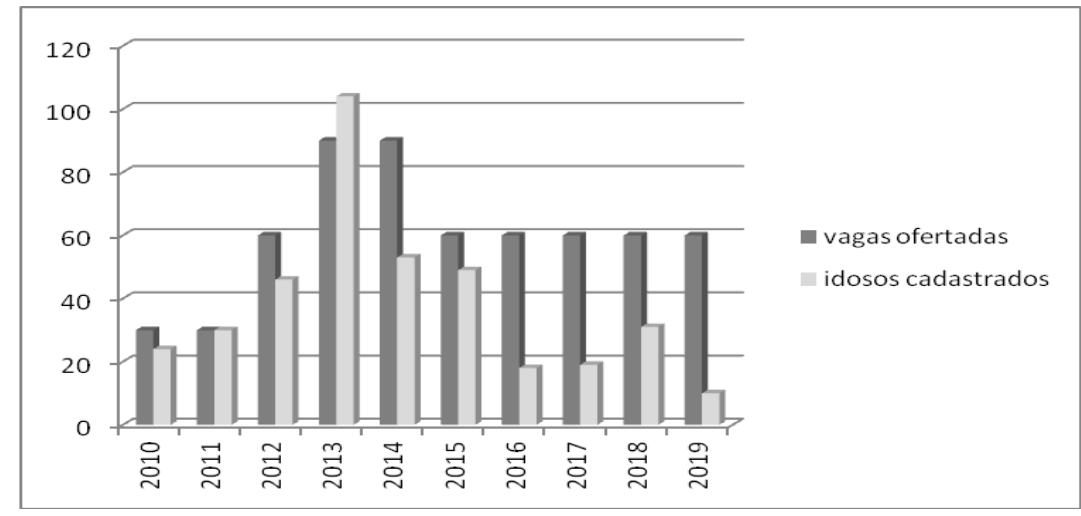

Figura1- Distribuição das vagas ofertadas e número de alunos inscritos no projeto Incluir entre 2010 e 2018, N= 384, Niterói, 2019.

A média de idade foi de 62,9 anos $(\mathrm{dp}=7,2)$, com maior número de mulheres (80\%), o que é coerente com o perfil dos participantes do programa de extensão Espaço Avançado, onde se dá a divulgação do curso.

A distribuição dos alunos nos módulos do curso é apresentada na figura 2. A grande maioria participou do módulo básico (49,2\%), seguida do módulo Avançado I (43,8). A média de inscritos por turma foi de aproximadamente 19 alunos $(d p=6,4)$. A equipe do projeto também variou ao longo dos anos, como pode ser observado na figura 3. Os instrutores, bolsistas ou voluntários, eram alunos do curso de Ciência da Computação e do curso de Sistemas de Informação da UFF. 


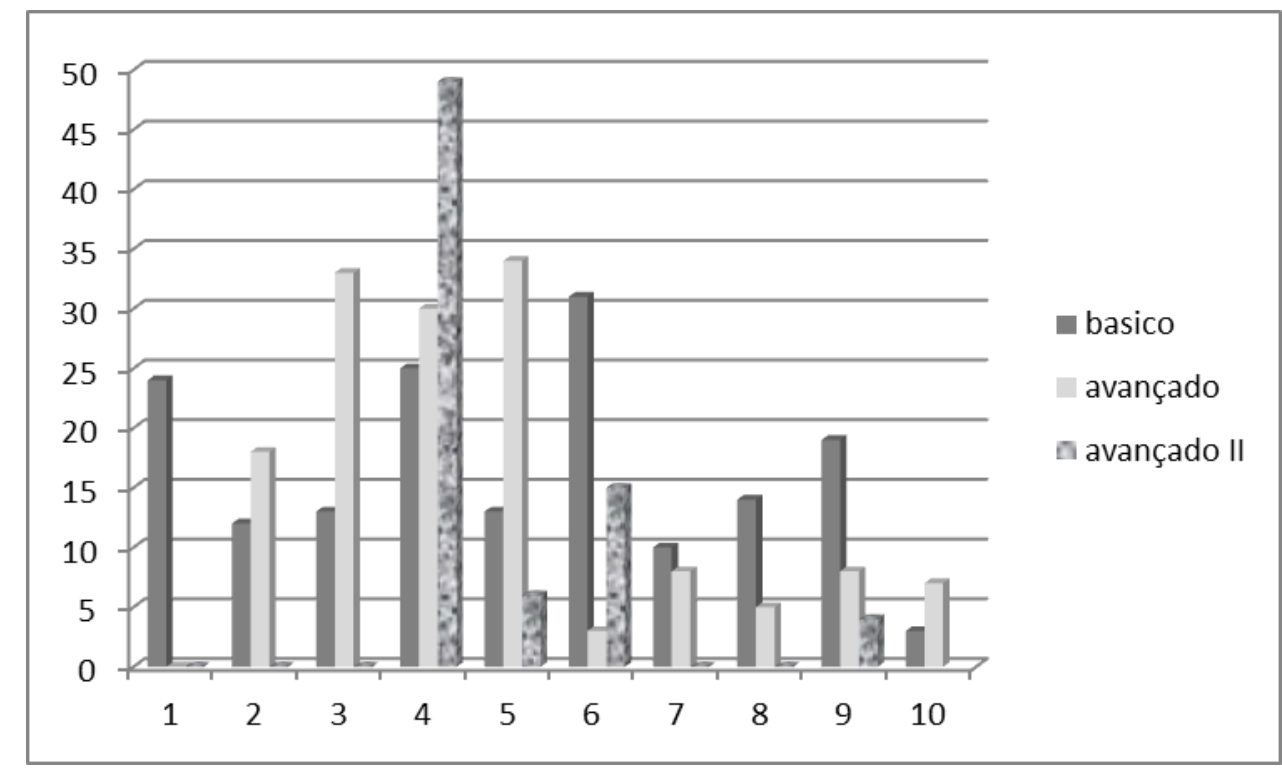

Figura 2 - Distribuição dos alunos quanto ao módulo cursado, N=384, Niterói, 2019

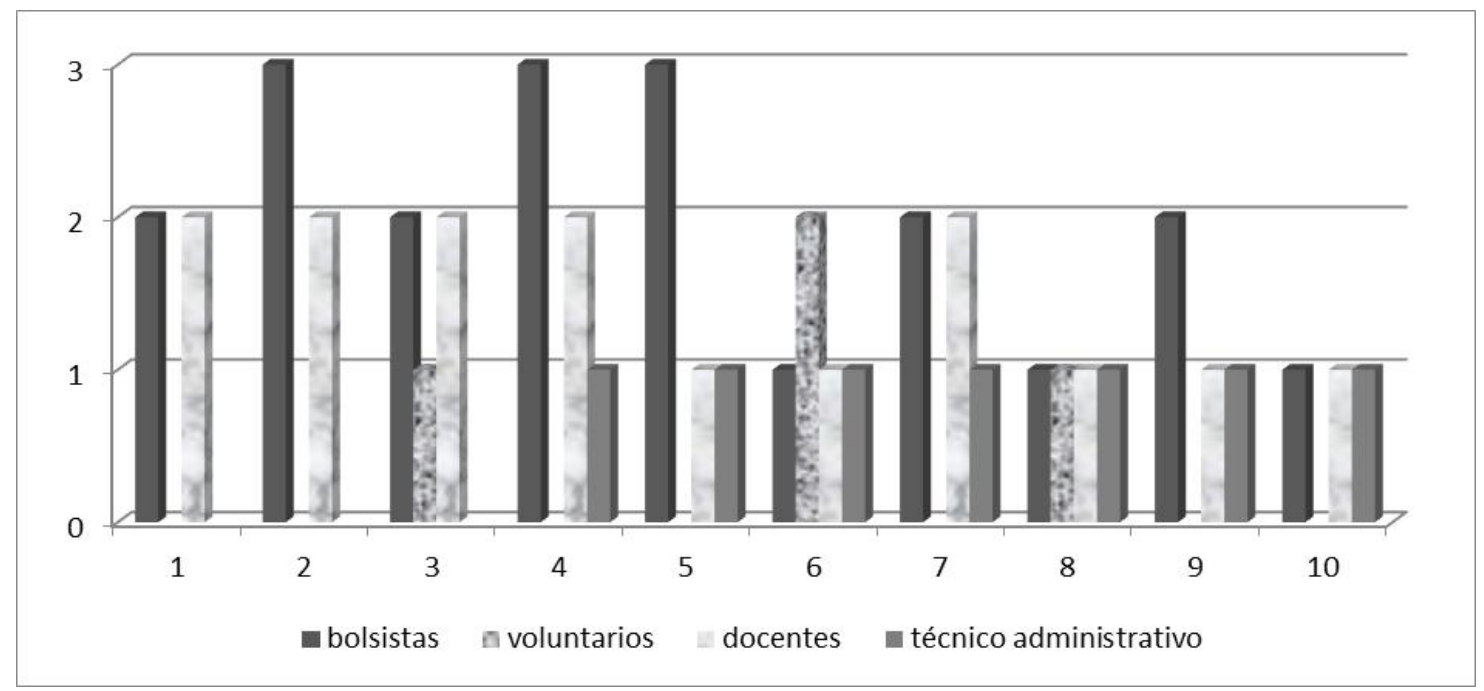

Figura 3 - Composição anual da equipe do projeto ao longo dos anos, Niterói, 2019.

A frequência média nas aulas foi de $92,63 \%(\mathrm{dp}=2,72)$ e apenas $8,3 \%$ dos alunos abandonaram o curso. A sugestão mais frequente (43\%) nos relatórios de avaliação do curso foi a de que as aulas ocorressem mais de uma vez por semana, visando melhorar a fixação dos conteúdos, seguida do desenvolvimento de material de suporte para atividades em casa (18\%). Outra questão identificada por $22,8 \%$ dos alunos foi o fato de não 
possuírem computador em casa, o que dificultava a fixação dos comandos e conteúdos apresentados. Alguns alunos também consideraram que o tempo de aula era muito pequeno (4\%).

Entre os temas centrais da avaliação encontram-se a satisfação com o curso, o aprendizado e o ganho de autonomia no uso de ferramentas de informática e na navegação na internet (Figura 4).

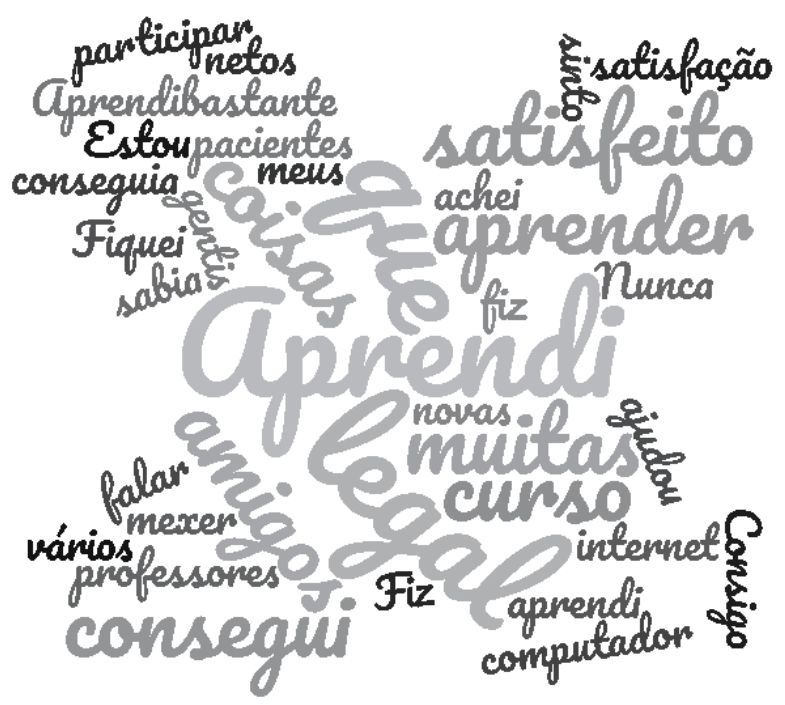

Figura 4 - Nuvem de palavras obtida a partir das avaliações dos alunos, N=88, Niterói, 2019.

O projeto tem participado da Semana de Extensão da UFF desde sua criação, tendo sido apresentados 13 trabalhos. Foi agraciado 2 vezes com o primeiro lugar e $2 \mathrm{com}$ o segundo lugar no Prêmio de Extensão Josué de Castro na área de Tecnologia (Figura 5).
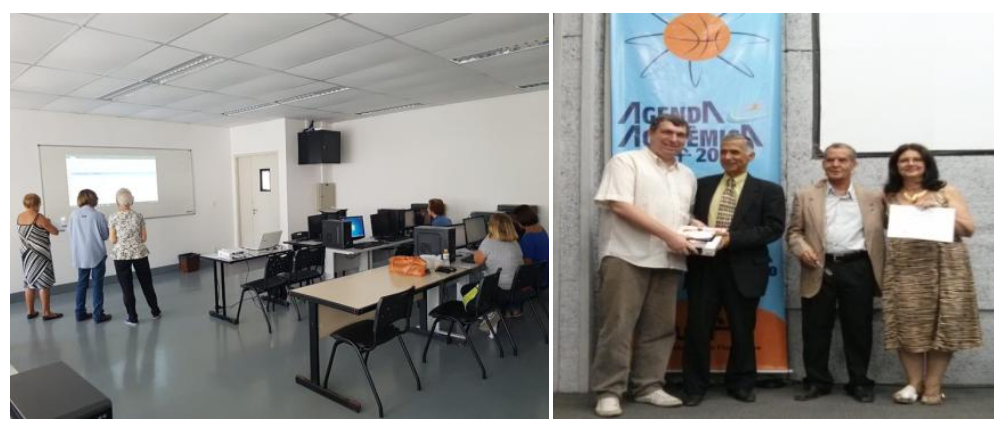
Figura 5 - (a) Foto de aula; (b) Recebimento pelo coordenador do projeto do 1o Lugar no XI Prêmio de Extensão Josué de Castro, 2013, na área de tecnologia.

\section{Discussão}

A estrutura do curso, em módulos de crescente complexidade, bem como os conteúdos trabalhados, estão de acordo com a estratégia adotada por outros cursos dedicados à inclusão digital de idosos. ${ }^{7,911,19}$ A maioria de alunos do sexo feminino e a média de idade dos alunos também se assemelham ao observado em outros projetos dedicados a este objetivo. ${ }^{7,14}$

A literatura aponta que a disponibilidade de tempo mais flexível para a execução das atividades é um elemento importante para o ensino de idosos. ${ }^{15}$ Embora a duração total do curso seja superior ao de outras experiências, ${ }^{9,11,20}$ é percebida pelos participantes como pequena, tendo sido registrada a demanda por mais tempo nas avaliações dos alunos. Na literatura há relato de experiência com mais horas de aula por semana. ${ }^{9}$ Vale ressaltar que a falta de computador em casa para treinar os conceitos apresentados nas aulas também foi apontada por alguns idosos. Este aspecto é um desafio para o projeto uma vez que a instituição não dispõe de um laboratório de informática específico para atividades de extensão. Desta forma, tanto a oferta de cursos com maior duração quanto à disponibilização do laboratório em outros horários para a prática dos conceitos apresentados pelos alunos ficam dificultadas.

Vale ressaltar que a indisponibilidade de computador nas residências e a possível descontinuidade de uso desta ferramenta, principais dificuldades indicadas pelos alunos, são apontadas por Barroso e $\mathrm{Maia}^{7}$ como fatores que podem contribuir para o esquecimento e a perda das habilidades adquiridas ao longo do curso.

O tamanho das turmas é outro fator descrito como importante para o ensino de idosos. ${ }^{15}$ Embora semelhante ao de outros projetos, ${ }^{7,9,20}$ a média de 19 alunos por turma 
dificulta, em alguns momentos, o atendimento mais individualizado das necessidades dos alunos, o que poderia ser importante sobretudo nas turmas do módulo básico. No entanto, o tamanho das turmas não tem se mostrado prejudicial à socialização dos participantes nem à sua satisfação com o curso. Outro aspecto que tem sido positivo é a homogeneidade na idade dos alunos, o que colabora tanto com a troca de experiências entre eles quanto com o planejamento das atividades em sala de aula. A baixa variação na idade dos alunos é considerada fator importante ao se tratar de educação de idosos. ${ }^{16}$

Soares e colaboradores ${ }^{11}$ avaliaram a experiência de oficinas de introdução à informática em associação a treino cognitivo para idosos na Bahia. Entre os benefícios observados pelos autores encontram-se ganhos no aprendizado do uso do computador, melhoria no humor e na integração dos idosos, principalmente com as gerações mais jovens. Barbosa e Maia ${ }^{7}$ também observaram evolução dos alunos na utilização dos computadores ao longo dos módulos de ensino. No entanto, as autoras ressaltam que muitos alunos demonstraram a intenção de aprimorar seus conhecimentos. Esses resultados são compatíveis com o observado na análise do discurso dos alunos do projeto Incluir.

O ganho de autonomia no uso de ferramentas de informática e na navegação na internet apontado pelos alunos sugere que o curso esteja contribuindo para sua inclusão social, sobretudo em função do crescimento na utilização das redes sociais no Brasil e seu potencial para aprimorar as relações sociais dos idosos. ${ }^{19}$

\section{Conclusão}

Os resultados permitem considerar que o Projeto Incluir vem cumprindo seus objetivos e oferece um espaço importante para a inclusão dos idosos atendidos no mundo da informática. No entanto, há ainda desafios a serem enfrentados como a compatibilização entre a disponibilidade de horário nos laboratórios do Instituto de Computação e a intenção de ofertar mais aulas na mesma semana e o aumento da duração de cada aula. Outro aspecto a ser considerado é a busca de novos mecanismos de 
divulgação do projeto, tendo em vista a existência de vagas que não vêm sendo efetivamente ocupadas nas diferentes turmas.

\section{Contribuições individuais dos autores}

José Raphael Bokehi é coordenador do projeto, participou da concepção e definição da metodologia do trabalho, análise dos dados, redação do artigo.

Gian Vitor Almeida Rocha é bolsista de extensão do projeto, participou da coleta de dados, análise dos dados e redação do artigo.

\section{Agradecimentos}

Os autores agradecem à PROEX/UFF pela concessão das bolsas de extensão.

\section{Referências}

1- DOS SANTOS, Lucimara Bianchin; ALEGRE, Animari Mayer; FREIRE, Emerson. Da inclusão digital à social: Um estudo a partir da experiência com idosos e adultos na FATEC Jundiaí. Fasci-Tech, São Caetano do Sul, v.1, n. 1, p. 114-125, Ago./Dez. 2009.

2 - ARAÚJO, Larissa Fortunato; COELHO, Carolina Gomes; DE MENDONÇA, Erica Toledo; VAZ, Aline Vasconcelos Martins; SIQUEIRA-BATISTA, Rodrigo; COTTA, Rosângela Minardi Mitre. Evidências da contribuição dos programas de assistência ao idoso na promoção do envelhecimento saudável no Brasil. Rev Panam Salud Publica, Panamá, v.30, n.1, p. 80-6, 2011.

3 - GEIB, Lorena Teresinha Consalter. Determinantes sociais da saúde do idoso. Ciência \& Saúde Coletiva, Rio de Janeiro, v.17, n. 1, p. 123-133, 2012. 
4 - VICENTE, Fernanda Regina; DOS SANTOS, Silvia Maria Azevedo. Avaliação multidimensional dos determinantes do envelhecimento ativo em idosos de um município de santa Catarina. Texto Contexto Enferm, Florianópolis, v. 22, n. 2, p. 370-8, Abr./Jun. 2013.

5 - DA SILVEIRA, Michele Marinho; ROCHA, Josemara de Paula; KÜMPEL, Daiana Argenta; WIBELINGER, Lia Mara; PASQUALOTTI, Adriano; COLUSSI, Eliane Lucia. Ambientes de aprendizagem: significado na vida de idosos frequentadores de oficinas de informática. Renote, Porto Alegre, v. 9, n. 1, p. 1-9, jul. 2011.

6 - TEZZA, Rafael; BONIA, Antonio Cezar. O idoso e a internet: uma etnografia sobre interação e aprendizagem. Perspectivas em Ciência da Informação, Belo Horizonte, v. 15, n. 1, p. 185-197, Jan./Abr. 2010.

7 - BARROSO Magali Maria de Araújo; MAIA, Miriam Lourenço. Inclusão digital na terceira idade. Revista Diálogos, Garanhuns, v.10, p. 76-85. 2008.

8 - SORJ, Bernardo; GUEDES, Luís Eduardo. Exclusão digital: problemas conceituais, evidências empíricas e políticas públicas. Novos estud. - CEBRAP, São Paulo, n. 72, p. 101-117, https://doi.org/10.1590/S0101-33002005000200006, 2005

9 -BIZELLI, Maria Helena Sebastiana Sahão; BARROZO, Sidineia; TANAKA, Julia Sawaki, SANDRON, Daniela Corsino. Informática para a terceira idade - características de um curso bem sucedido. Rev. Ciênc. Ext., São Paulo, v.5, n.2, p.4-14, 2009.

10 - SANTANA, Áureo Evangelista; LEITE, Jacqueline Nayara Fereraça; COELHO, Stefanie Bertti; FIGUEIRA, Mathilde Elisa. Universidade aberta à terceira idade: paradigma de um ambiente inclusivo e intergeracional. Rev. Ciênc. Ext., São Paulo, v.7, n.2, p. 160, 2011. 
11 - SOARES, Zelma Freitas; DE SANTANA, Elisangela Ferreira; RABELO, Dóris Firmino. Iniciação à informática associada ao treino cognitivo com idosos. Rev. Ciênc. Ext., São Paulo, v.11, n.3, p.155-167, 2015.

12 - KERÄNEN, Nina Susanna; KANGAS, Maarit; IMMONEN, Milla; SIMILÄ, Heidi; ENWALD, Heidi; KORPELAINEN, Raija; JÄMSÄ, Timo. Use of Information and Communication Technologies Among Older People With and Without Frailty: A Population-Based Survey. J Med Internet Res., v.19, n. 2; e29. doi: 10.2196/jmir.5507, 2017.

13 - KRUG, Rodrigo de Rosso; XAVIER, André Junqueira; D’ORSI, Eleonora. Factors associated with maintenance of the use of internet, EpiFloripa Idoso longitudinal study. Rev. Saúde Pública, São Paulo, v. 52, n. 37, 2018. Available from $<$ http://www.scielo.br/scielo.php?script=sci_arttext\&pid=S0034-

89102018000100230\&lng=en\& $n$ rm=iso>. access on 02 May 2019. Epub Apr 09, 2018. http://dx.doi.org/10.11606/s1518-8787.2018052000216.

14 - LOLLI, Maria Carolina Gobbi dos Santos; MAIO, Eliane Rose. Uso da tecnologia por idosos: perfil, motivações, interesses e dificuldades. Revista ECS, Sinop, v. 5, n. 2, p. 211-223, 2015.

15 - OLIVEIRA, Karoline Leite Guedes. Idosos em rede: a construção de novas práticas culturais mediadas por tecnologias. 2013. 159 f. Dissertação (Mestrado em Educação)Universidade Federal do Rio Grande do Sul, Porto Alegre, 2013.

16 - BATISTA, Marina Picazzio Perez; SOUZA, Fabiana Gomes; SCHWARTZ, Gilson; EXNER, Camila; ALMEIDA, Maria Helena Morgani. Utilização no cotidiano de tecnologias da informação e comunicação por idosos participantes da Universidade Aberta da Terceira Idade da Universidade de São Paulo. Revista Kairós Gerontologia, São Paulo, v.18, n. 4, p. 405-426, 2015. 
17- GROSSI, Márcia Gorett Ribeiro; COSTA, José Wilson; SANTOS, Ademir José. A exclusão digital: o reflexo da desigualdade social no Brasil. Nuances: estudos sobre Educação, Presidente Prudente, v.24, n.2, p. 68-85, 2013.

18. BARDIN, Laurence. Análise de conteúdo, Lisboa: Edições 70, 2010, 281 p.

19. CHEPE, Lucélia Moreira; ADAMATTI, Diana Francisca. Estudo Sobre Interação de Idosos em Redes Sociais Digitais. Informática na Educação: teoria e prática, Porto Alegre, v. 18, n. 2, p. 177-198, jul./dez. 2015.

20. MACHADO, Letícia Rocha; GRANDE, Tássia Priscila Fagundes; BEHAR, Patricia Alejandra; LUNA, Fabiana de Miranda Rocha. Mapeamento de competências digitais: a inclusão social dos idosos. Educ. Temat. Digit., Campinas, v 18, n 4, p. 903-921. 2016. 